\title{
Commentary: A neural marker of medical visual expertise
}

\author{
Liam Rourke ${ }^{1 *}$, Ron Damant ${ }^{1}$, Chris Donoff ${ }^{2}$, Anthony Singhal ${ }^{2}$ \\ 'Department of Medicine, University of Alberta, Edmonton, Canada \\ ${ }^{2}$ Department of Psychology, University of Alberta, Edmonton, Canada
}

Article Info

\section{Article Notes}

Received: March 13, 2018

Accepted: April 19, 2018

\section{*Correspondence:}

Dr. Liam Rourke, Department of Medicine, University of Alberta, Edmonton, Canada;

Email: liam.rourke@gmail.com

(c) 2018 Rourke L. This article is distributed under the terms of the Creative Commons Attribution 4.0 International License.
Recently we identified a neural marker of medical visual expertise, that is, a distinctive pattern of brain activity that appears when a specialist reads images from her area of expertise ${ }^{1}$. The timing and location of the activity points to a conspicuous but neglected aspect of diagnostic image interpretation: it draws not only on conceptual knowledge and clinical reasoning, but also vision. Pulmonology educators should incorporate this fact in the education of their trainees. Decades of research on superior performance demonstrate how perceptual expertise develops and how it is trained across several domains. The eclectic group of experts who have been studied include fingerprint analysts, chicken sexers, birders, and lepidopterists, among others, and too rarely, medical specialists such as radiologists and the pulmonologists and cardiologists that we studied ${ }^{2-6}$. The models and evidence emerging from this field should guide instructional designers in the same way that models and evidence of cognition guide the instruction of conceptual knowledge and clinical reasoning. Currently, they do not. In this commentary we introduce some of the frameworks that have shaped research on perceptual expertise, and we summarize relevant evidence. Our discussion extends to the three senses relevant for pulmonary diagnosis: seeing, hearing, and touching.

One way that educators improve medical training is by studying experts-those who demonstrate consistently superior performance in a domain relative to other domains and relative to novices ${ }^{7}$. Educators identify the components of expert performance, and these become curricular foci. Perceptual abilities are sometimes among these components. For instance, the competency statement developed by the American College of Cardiology specifies the minimum knowledge necessary for competence in interpreting ECGs. It reads: "Electrocardiographic interpretation requires a basic knowledge of electrocardiographic technology, cardiac anatomy and electrophysiology, and the ability to recognize diagnostic patterns on a 12-lead tracing ${ }^{8}$. This imperative, notably, emerged from their review of the literature which concluded that multiple cardiologists reading the same ECG often vary substantially, and further, one cardiologist reading the same ECG on multiple occasions can have substantively different interpretations ${ }^{9}$.

For some tasks, superior means of perceiving and processing information are the things that separate experts from novices, and this gives rise to the term perceptual expertise. Perceptual experts are those who are able to make fine distinctions among perceptually 
similar stimuli consistently and accurately. Across several decades, researchers have examined experts in gustation, active touch, audition, and vision, including sommeliers, readers of braille, fretted instrument players, and the sample of visual experts we listed previously ${ }^{2-6,}$ 10-12. Together this research is culminating in a rich understanding of the behavioral, cognitive, and neural components of perceptual expertise.

Pulmonologists routinely enact multiple forms of perceptual expertise in their practice. Auditory expertise is required to detect adventitious lung sounds during auscultation or to categorize a crackle as fine or coarse and thereby diagnose pneumonia as acute or resolving. Tactile expertise is required to assess the vibrations, asymmetries, and excursions evoked through palpation and to determine whether a stimulus is suggestive of a malignancy, COPD, or pneumonia. Visual expertise is required to decode the wide variety of imaging studies available to pulmonologists. Diagnostic sounds, sensations, and sights are complex stimuli in which the difference between normal and abnormal findings is subtle. Interpreting them correctly is a difficult task that exemplifies expertise.

We gathered evidence for our claim that the visual expertise exercised by pulmonologists is similar, neurophysiologically, to other forms of visual expertise ${ }^{1}$. We recorded the brain activity of pulmonologists and cardiologists as they categorized images of chest x-rays indicating lung disease (the pulmonologists' expertise) and EKGs indicating common cardiovascular diagnoses (the cardiologists' expertise). Our findings replicated those of researchers who have studied visual experts using this paradigm: The pattern of electrophysiological activity is qualitatively different when specialists examine images from their area of expertise than when they examine images for which they are simply competent. Different cortical pathways; comprised of neurons tuned to the pertinent tactile, auditory, and visual features of diagnostic stimuli; engaged in different types of processing, develop to process the stimuli that a specialist encounters regularly.

Does this mean that pulmonologists do not draw on their knowledge of anatomy, physiology, and the biological bases of disease when they are interpreting diagnostic stimuli; that their eye, ear, or hand is not guided by a particular hypothesis they are investigating, one based on a patient's history, clinical presentation, and lab valuesor a pattern among all three? The question is contentious. Scientists of vision, cognition, and neuroscience, in turns, present persuasive evidence that i) perception is directed at every stage by conceptual knowledge and the perceiver's goals, or that ii) perception is unaffected by knowledge and goals, or that iii) perception is a discrete stage of processing that subsequently combines with conceptual knowledge to inform interpretation ${ }^{13-16}$. The purpose of this commentary is not to resolve that question. Instead, it is to show pulmonology educators that there is a perceptual component to interpreting diagnostic stimuli, and to urge them to address this in the curriculum.

There is a sophisticated body of theory and evidence on the development, training, and assessment of perceptual expertise, and our study provides a bridge from that literature to medical education. Perceptual expertise develops through years of consequential experience with a particular category of stimuli. Improvement in performance is instantiated at the neural level, and adaptations include the densification of neurons representing the stimuli, the tuning of these neurons' receptive fields to the relevant features of the stimuli, and the appropriation of neural circuits specialized for complicated perceptual judgments.

Researchers have documented these changes by studying existing experts, but, perhaps more useful to medical educators, they also train participants to become perceptual experts. The approach is epitomized in the germinal work of Isabell Gauthier ${ }^{17}$. Stimuli are gathered that exemplify diagnostic features that differentiate findings. The stimuli are presented to trainees, first with labels (e.g., hyperresonant versus dull percussion sounds or fine versus coarse lung sounds), and second, without labels so that trainees can provide them. Feedback is then provided on the accuracy of the trainees categorizations. Training begins with gross distinctions and progresses to the subtle categorizations that characterize expertise as participants encounter hundreds of stimuli and make decisions with feedback.

We employed this straightforward procedure to train participants to discriminate harmless from suspicious skin lesions ${ }^{18}$. We were able to improve the ability of undergraduate psychology students-participants with no medical training and therefore little conceptual knowledge of the pathophysiology of the skin or clinical reasoning ability-to distinguish harmless from suspicious skin lesions. At the outset of the study, our participants' ability to discriminate benign lesions from melanomas was less than chance; at the conclusion of training it was 95\%. Their response bias response time, and sensitivity also improved substantially. This improvement was accomplished in four training sessions, spaced across four days, with each session lasting approximately 30 -minutes. The improvements are remarkable given that the lesions we used in our post-test were not the images used in training or in our pre-test-a issue of design which is rarely followed in similar studies. The structured discrimination training distills months of clinical experience received during rotations into a much shorter duration and perhaps mimics the volume of experience a clinician acquires over years of practice.

Over the last several decades, medical educators have 
become increasingly knowledgeable about educational theory and its empirical findings, and this knowledge has improved teaching practices. However, it has not improved the way that students and residents learn to interpret the diagnostic stimuli they gather through their senses. This is unfortunate. Perceptual expertise and perceptual learning are subjects of a robust body of literature that could enhance the acquisition of skills that are central to clinical practice. Traditionally, instructors have focused on imparting the conceptual knowledge, clinical reasoning, and procedural skills that underlie diagnosis. Each component is important; however, they are not more important that the tactile, auditory, and visual skills that are at the center of these diagnostic events. Researchers in the cognitive neurosciences have been successfully imparting perceptual skills for decades, and they have refined methods for doing so. Pulmonology educators should adopt these methods.

\section{References}

1. Rourke L, Cruikshank LC, Shapke L, et al. A neural marker of medical visual expertise: implications for training. Advances in Health Sciences Education. 2016; 21(5): 953-966.

2. Emerick B, Vanderkolk J, Busey T. The Policy Implications of Research on Fingerprint Examination Tasks. Policy Insights from the Behavioral and Brain Sciences. 2015; 2(1): 166-174.

3. Biederman I, Shiffrar MM. Sexing day-old chicks: A case study and expert systems analysis of a difficult perceptual-learning task. Journal of Experimental Psychology: Learning, memory, and cognition. 1987; 13(4): 640.

4. Gauthier I, Skudlarski P, Gore JC, et al. Expertise for cars and birds recruits brain areas involved in face recognition. Nat Neurosci. 2000; 3: 191-197 10.1038/72140

5. Rhodes G, Byatt G, Michie PT, et al. Is the fusiform face area specialized for faces, individuation, or expert individuation. Journal of cognitive neuroscience. 2004; 16(2): 189-203.

6. Harley EM, Pope WB, Villablanca JP, et al. Engagement of fusiform cortex and disengagement of Harel, A., Kravitz, D., \& Baker, C. I. (2013). Beyond perceptual expertise: revisiting the neural substrates of expert object recognition. Frontiers in human neuroscience. 2009; 7: 885 .

7. Ericsson KA, Hoffman RR, KozbeltA, etal. (Eds.). The Cambridge handbook of expertise and expert performance. Cambridge University Press. 2018.

8. Kadish AH, Buxton AE, Kennedy HL, et al. ACC/AHA clinical competence statement on electrocardiography and ambulatory electrocardiography: A report of the ACC/AHA/ACP-ASIM Task Force on Clinical Competence (ACC/AHA Committee to Develop a Clinical Competence Statement on Electrocardiography and Ambulatory Electrocardiography) Endorsed by the International Society for Holter and Noninvasive Electrocardiology. Circulation. 2001; 104(25): 3169-3178.

9. Salerno SM, Alguire PC, Waxman HS. Competency in interpretation of 12-lead electrocardiograms: a summary and appraisal of published evidence. Annals of Internal Medicine. 2003; 138(9): 751-760.

10. Castriota-Scanderbeg A, Hagberg GE, Cerasa A, et al. The appreciation of wine by sommeliers: a functional magnetic resonance study of sensory integration. Neuroimage. 2005; 25(2): 570-578.

11. Behrmann M, Ewell C. Expertise in tactile pattern recognition. Psychological Science. 2003; 14(5): 480-492.

12. Strait DL, Kraus N. Biological impact of auditory expertise across the life span: musicians as a model of auditory learning. Hearing research. 2014; 308: 109-121.

13. Collins JA, Olson IR. Knowledge is power: How conceptual knowledge transforms visual cognition. Psychonomic Bulletin \& Review. 2014; 21(4): 843-860.

14. Harel A, Kravitz D, Baker CI. Beyond perceptual expertise: revisiting the neural substrates of expert object recognition. Frontiers in human neuroscience. . 2013; 7: 885.

15. Pylyshyn Z. Is vision continuous with cognition?: The case for cognitive impenetrability of visual perception. Behavioral and brain sciences. 1999; 22(3): 341-365.

16. Poggio T, Ullman S. Vision: are models of object recognition catching up with the brain?. Annals of the New York Academy of Sciences. 2013; 1305(1): 72-82.

17. Gauthier I, Tarr MJ. Becoming a "Greeble" expert: Exploring mechanisms for face recognition. Vision research. 1997; 37(12): 1673-1682.

18. Xu Y. Revisiting the role of the fusiform face area in visual expertise. Cerebral Cortex. 2005; 15(8): 1234-1242. 